\title{
Clinical utility gene card for: Dyskeratosis congenita - update 2015
}

\author{
Inderjeet Dokal $^{\star, 1}$, Tom Vulliamy ${ }^{1}$, Philip Mason ${ }^{2}$ and Monica Bessler ${ }^{2}$ \\ European Journal of Human Genetics (2015) 23, doi:10.1038/ejhg.2014.170; published online 3 September 2014 \\ Update to: European Journal of Human Genetics (2011) 19, doi:10.1038/ejhg.2011.90; published online 25 May 2011
}

\section{DISEASE CHARACTERISTICS}

1.1 Name of the disease (synonyms)

Dyskeratosis congenita (Zinsser-Engman-Cole Syndrome). ${ }^{1-11}$

1.2 OMIM\# of the disease

Three modes of inheritance:

\section{X-linked recessive}

Autosomal dominant

(OMIM \#305000)

Autosomal recessive

(OMIM \#127550)

(OMIM \#224230)

1.3 Name of the analysed genes or DNA/chromosome segments

\begin{tabular}{lll}
\hline DKC1 & X-linked recessive & $(\sim 25 \%$ of DC) \\
TERC & Autosomal dominant & $(\sim 5 \%$ of DC) \\
TERT & Autosomal dominant and autosomal recessive & $(\sim 5 \%$ of DC) \\
NOP10 & Autosomal recessive & $(<1 \%$ of DC) \\
NHP2 & Autosomal recessive & $(<1 \%$ of DC) \\
TINF2 & Autosomal dominant & $(\sim 12 \%$ of DC) \\
USB1 & Autosomal recessive & $(\sim 2 \%$ of DC) \\
TCAB1 & Autosomal recessive & $(<1 \%$ of DC) \\
CTC1 & Autosomal recessive & $(\sim 1 \%$ of DC) \\
RTEL1 & Autosomal recessive & $(\sim 2 \%$ of DC) \\
\hline
\end{tabular}

\subsection{OMIM\# of the gene(s)}

\section{$D K C 1$}

TERC

TERT

NOP10

NHP2

TINF2

USB1

TCAB 1

CTC1

RTEL 1
OMIM \#300126 OMIM \#602322 OMIM \#613989 OMIM \#606471 OMIM \#606470 OMIM \#604319 OMIM \#613276 OMIM \#612661 OMIM \#613129 OMIM \#608833
1.5 Mutational spectrum

(Supplementary Table S1 and http://telomerase.asu.edu/diseases.html)

DKC1 Mainly missense variants, $>50$ different variants reported

TERC Heterozygous variants - point variants, small and large deletions, >40 different variants reported

TERT Heterozygous and biallelic variants. Mainly missense variants, $>50$ different variants reported

NOP10 One homozygous missense variant

NHP2 Biallelic variants reported in two families

TINF2 Mainly missense variants, $>20$ different variants reported, often de-novo.

USB1 Homozygous or biallelic frameshift, nonsense or splice site variants; 10 different variants reported

TCAB1 Biallelic variants reported in two families

CTC1 Biallelic variants reported in five families

RTEL1 Biallelic variants reported in 11 families

\subsection{Analytical methods}

PCR amplification of genomic DNA fragment(s) of DC genes (DKC1, TERC, TERT, NOP10, NHP2, TINF2, USB1, TCAB1, CTC1 and RTEL1) followed by denaturing, HPLC and/or direct DNA sequence analysis.

\subsection{Analytical validation}

Sequencing of the appropriate DNA fragment.

1.8 Estimated frequency of the disease

(Incidence at birth ('birth prevalence') or population prevalence):

1 in 1000000 (approximate).

1.9 If applicable, prevalence in the ethnic group of investigated person 1 in 1000000 (approximate).

1.10 Diagnostic setting

\begin{tabular}{lcc}
\hline & Yes & No \\
A. (Differential) diagnostics & $\bigotimes$ & $\square$ \\
B. Predictive testing & $\bigotimes$ & $\square$ \\
C. Risk assessment in relatives & $\bigotimes$ & $\square$ \\
D. Prenatal & $\bigotimes$ & $\square$
\end{tabular}

\footnotetext{
${ }^{1}$ Centre for Paediatrics, Blizard Institute, Queen Mary University of London, Barts and the London School of Medicine and Dentistry, Whitechapel, London, UK; ${ }^{2}$ Paediatric Research Center, The Children's Hospital of Philadelphia, Philadelphia, PA, USA

${ }^{*}$ Correspondence: Professor I Dokal, Centre for Paediatrics, Blizard Institute, Queen Mary University of London, Barts and the London School of Medicine and Dentistry, Whitechapel, 4 Newark Street, Whitechapel, London E1 2AT, UK. Tel: + 44 (0) 207882 2205; Fax: + 44 (0) 207882 2195; E-mail: i.dokal@qmul.ac.uk

Received 14 May 2014; revised 11 July 2014; accepted 30 July 2014
} 
Comment: Dyskeratosis congenita (DC) is an inherited bone marrow (BM) failure syndrome exhibiting considerable clinical and genetic heterogeneity. In its classical form DC is characterized by a mucocutaneous triad of abnormal skin pigmentation, nail dystrophy, and mucosal leucoplakia. A given patient may also have a variety of other somatic features and there is an increased risk of malignancy. $\mathrm{X}$-linked recessive, autosomal dominant $(\mathrm{AD})$, and autosomal recessive (AR) forms of DC are recognised. The gene mutated in X-linked DC (DKC1) encodes a highly conserved nucleolar protein called dyskerin. Dyskerin associates with the H/ACA class of small nucleolar RNAs in small nucleolar ribonucleoprotein particles (snoRNPs), which are important in guiding the conversion of uracil to pseudouracil during the maturation of ribosomal RNA. Dyskerin also associates with the RNA component of telomerase (TERC) where it is important in stabilising the telomerase complex, which is critical in the maintenance of telomeres. Heterozygous variants in TERC (telomerase RNA component) and TERT (telomerase reverse transcriptase component) have been found in patients with $\mathrm{AD}-\mathrm{DC}$ and in some patients with aplastic anaemia (AA), myelodysplasia (MDS), and idiopathic pulmonary fibrosis. One AD-DC subtype was found to be due to variants in TINF2, which encodes a component of the shelterin complex that protects telomeres and controls access to the telomere. TINF2 variants can sometimes be present with AA. It has also been established that AR-DC is genetically heterogeneous with seven characterized subtypes owing to biallelic variants in NHP2, NOP10, TERT, USB1, TCAB1, CTC1, and RTEL1. A subset of patients with the multisystem disorders Hoyeraal-Hreidarsson $(\mathrm{HH})$ and Revesz (RS) syndromes have been found to have DKC1, TINF2, or RTEL1 variants. Collectively, these findings have demonstrated that classical DC, HH, RS, a subset of AA, MDS/ AML and pulmonary fibrosis are principally due to defects in telomere maintenance and cells from these patients usually have short/abnormal telomeres (except patients with USB1 mutations). Equally it has become clear that the spectrum of patients who can now be considered to have DC, has expanded considerably. This does raise the issue as to what should now be called DC and it has become increasingly popular to refer to them as 'the telomereopathies' or 'short telomere syndromes' or 'telomere biology disorders'.

The following categories of individuals can be considered to have DC:

1. Those with all three (abnormal skin pigmentation, nail dystrophy, and leucoplakia) mucocutaneous features.

2. Individuals with 1 out of 3 mucocutaneous features, $+\mathrm{BM}$ failure +2 other somatic features of DC.

3. Those presenting with AA or MDS or pulmonary fibrosis associated with a pathogenic telomerase variant.

4. Individuals having four or more of features of the HoyeraalHreidarsson syndrome (growth retardation, developmental delay, microcephaly, BM failure, immunodeficiency, and cerebellar hypoplasia).

5. Individuals with two or more features seen in DC associated with very short telomeres $(<1$ st centile)

NB: It is noteworthy 'classical DC' often presents as a multisystem disorder in the paediatric age group, whereas adult patients presenting with one or more feature of DC display a very variable phenotype and the associated telomerase mutations are usually acting as risk factors. DC and related disorders thus represent a very wide clinical and genetic spectrum.

\section{TEST CHARACTERISTICS}

\begin{tabular}{|c|c|c|c|c|}
\hline & \multicolumn{2}{|c|}{ Genotype or disease } & \multirow{2}{*}{$\begin{array}{l}\text { A: True positives } \\
\text { B: False positives }\end{array}$} & \multirow{2}{*}{$\begin{array}{l}\text { C: False negative } \\
\text { D: True negative }\end{array}$} \\
\hline & Present & Absent & & \\
\hline \multicolumn{5}{|l|}{ Test } \\
\hline \multirow[t]{2}{*}{ Positive } & $A$ & $\mathrm{~B}$ & Sensitivity: & $A /(A+C)$ \\
\hline & & & Specificity: & $D /(D+B)$ \\
\hline \multirow[t]{2}{*}{ Negative } & C & D & Positive value: & $A /(A+B)$ \\
\hline & & & Negative value: & $\mathrm{D} /(\mathrm{C}+\mathrm{D})$ \\
\hline
\end{tabular}

\subsection{Analytical sensitivity}

(proportion of positive tests if the genotype is present)

Not determined; probably $>95 \%$.

\subsection{Analytical specificity}

(proportion of negative tests if the genotype is not present)

Difficult to comment on as the genetic basis is currently only known for approximately $60 \%$ of DC patients.

\subsection{Clinical sensitivity \\ (proportion of positive tests if the disease is present)}

The clinical sensitivity can be dependent on variable factors such as age or family history. In such cases a general statement should be given, even if a quantification can only be made case by case.

As features of the disease develop progressively with time individuals with pathogenic variants may not have any/all clinical features at the time of analysis. The age of onset and range of clinical features is very variable.

\subsection{Clinical specificity}

(proportion of negative tests if the disease is not present)

The clinical specificity can be dependent on variable factors such as age or family history. In such cases a general statement should be given, even if a quantification can only be made case by case.

As the genetic diagnosis can only be substantiated in about $60 \%$ of cases at present, not finding a variant in 1 of the 10 known $D C$ genes does not definitively exclude the diagnosis of DC.

\subsection{Positive clinical predictive value}

(life time risk to develop the disease if the test is positive)

This is high $(>90 \%)$ for DKC1 and TINF2 variants, but difficult to be precise for TERC and TERT variants, as variants in these genes have a variable penetrance, and are sometimes seen as risk factors for the development of disease.

\subsection{Negative clinical predictive value}

(probability not to develop the disease if the test is negative)

Assume an increased risk based on family history for a non-affected person. Allelic and locus heterogeneity may need to be considered.

Index case in that family had been tested:

Index case in that family had not been tested:

As highlighted previously, since the genetic basis is only known in about $60 \%$ of DC cases not finding a variant in 1 of the 10 known DC genes does not exclude the possibility of developing DC. It is also important to note that some mutations (particularly in the TERT gene) are not associated with disease in all individuals carrying the variant; suggesting that the variant is more a risk factor for the development of disease. 


\section{CLINICAL UTILITY}

3.1 (Differential) diagnosis: The tested person is clinically affected (To be answered if in 1.10 'A' was marked)

\subsubsection{Can a diagnosis be made other than through a genetic test?}

\begin{tabular}{ll}
\hline No & $\square$ (continue with \\
3.1.4) & \\
Yes & $\square$ \\
Clinically & \\
Imaging & $\square$ \\
Endoscopy & $\square$ \\
Biochemistry & $\square$ \\
Electrophysiology & $\square$ \\
Other (please & $\square$ Through identification of very short telomere length in \\
describe) & leucocytes(<1st centile) using Flow-FISH, Q-FISH, or \\
& quantitative PCR
\end{tabular}

\subsubsection{Describe the burden of alternative diagnostic methods to the} patient.

In patients with classical features (eg, presence of mucocutaneous triad), clinical diagnosis is possible. However as there is considerable variant in the onset and severity of clinical features, diagnosis exclusively based on clinical features is both difficult and unreliable. This is further highlighted by some of the 'cryptic' presentations of DC (eg, aplastic anaemia); in such cases it is the finding of a pathogenic variant in one of the $D C$ genes that helps in clinching the diagnosis of DC.

\subsubsection{How is the cost effectiveness of alternative diagnostic methods} to be judged?

Clinical diagnosis is dependent on clinical expertise. A delay in diagnosis can lead to inappropriate and inadequate management. This may, in some cases, lead to increased morbidity and mortality.

Flow-FISH and Q-FISH measurement of leucocyte telomere length are currently only available as a diagnostic method in a few labs and are relatively expensive. Telomere length measurement should perhaps be regarded as a good screening test for DC. Individuals found to have very short telomeres ( $<1$ st centile) should ideally go on to have genetic analysis for the $D C$ genes.

\subsubsection{Will disease management be influenced by the result of a} genetic test?

No $\square$

Yes $\square$

Therapy (please This is complicated. For the haematological defect describe)

Prognosis (please describe) (which is a major cause of mortality) patients with DC are more likely to respond to androgens (eg, oxymetholone or danazol) rather than to the conventional immunosuppressive (antithymocyte globulin and cyclosporine) therapy used in idiopathic aplastic anaemia. The diagnosis of DC will also influence the details of patient monitoring. It will also influence the drug conditioning regimen (low intensity) in patients who become candidates for BM transplantation.

This can vary considerably from death in infancy (usually due to bone marrow failure or immune deficiency) to that in the seventh decade. The major causes of death relate to BM failure ( $\sim 70 \%)$, cancer $(\sim 10 \%)$ and lung disease (pulmonary interstitial disease, $\sim 10$ $20 \%$ ). The BM failure develops progressively with time; up to $80 \%$ of patients will have BM failure by the age of 30 years. Cancer (haematological and nonhaematological) usually develops after the third decade. The most frequent solid malignancies are head and neck squamous cell carcinomas.

Management (please As this is a multisystem disorder it is important to describe) monitor many systems of the body. $\mathrm{BM}$ failure is one of the commonest and severe complications. About $50-70 \%$ of patients will respond to androgens, but patients have to be monitored carefully for side effects. For those who do not have an efficacious response to androgens and have a compatible BM donor, haemopoietic stem cell transplantation using a low-intensity conditioning regime is an option. It is important to use low-intensity conditioning regimes as conventional regimes using radiotherapy or busulphan are associated with high toxicity and poor survival. Treatment for cancer depends on the specific cancer but consideration has to be given to the underlying DC (ie, more supportive care, reduce drug doses).

With regards to pulmonary disease, patients should be encouraged to avoid smoking. Medical treatment is usually difficult in severe lung disease; lung transplant may be an option in some cases.

Advice on skin care (eg, use of moisturising creams) and avoidance of sunlight is important.

Liver disease (cirrhosis and noncirrhotic portal hypertension) is more common in DC patients than the normal population. Alcohol consumption should therefore be kept to a minimum and all drug administrations require close monitoring.

3.2 Predictive setting: The tested person is clinically unaffected but carries an increased risk based on family history

(To be answered if in 1.10 'B' was marked)

\subsubsection{Will the result of a genetic test influence lifestyle and} prevention?

If the test result is positive (please describe):

The management plan will have an impact on lifestyle. As highlighted above it is important that patients with DC (or predicted to be at risk of developing DC based on genetic testing) avoid smoking, sunlight, and keep alcohol intake to a minimum. They should also avoid occupations that expose them to hazardous chemicals.

If the test result is negative (please describe):

No specific lifestyle change necessary.

3.2.2 Which options in view of lifestyle and prevention does a person at-risk have if no genetic test has been done (please describe)?

Uncertainty will remain for the individual as to whether they need to modify their lifestyle with respect to smoking, alcohol, and sun exposure.

3.3 Genetic risk assessment in family members of a diseased person (To be answered if in 1.10 ' $\mathrm{C}$ ' was marked)

3.3.1 Does the result of a genetic test resolve the genetic situation in that family?

Yes, if a pathogenic mutation has been identified in a DC causing gene it is possible to offer genetic testing and appropriate counselling to all family members. 


\subsubsection{Can a genetic test in the index patient save genetic or other} tests in family members?

In some circumstances only. For example, in the case of TINF2 mutations, usually the mutation has arisen de-novo in the index case; it is therefore important to test the parents to establish this. In these families it then makes it unnecessary to test the unaffected sibs of the index case.

\subsubsection{Does a positive genetic test result in the index patient enable a predictive test in a family member?}

Yes. NB: There can be variability in disease expression even within families (suggesting a role of other genetic and/or environmental factors in the development of disease features). This always needs to be communicated to family members.

\subsection{Prenatal diagnosis}

(To be answered if in 1.10 'D' was marked)

\subsubsection{Does a positive genetic test result in the index patient enable a prenatal diagnosis?}

Yes.

\section{IF APPLICABLE, FURTHER CONSEQUENCES OF TESTING}

Please assume that the result of a genetic test has no immediate medical consequences. Is there any evidence that a genetic test is nevertheless useful for the patient or his/her relatives? (Please describe)

In female carriers of $\mathrm{X}$-linked DC (DKC1), there is usually no medical problem. However, X-linked DC carriers can go onto give rise to affected boys. Carrier testing for the $\mathrm{X}$-linked gene (DKC1) in appropriate families is therefore useful even though usually it has no immediate clinical consequences for the carrier.

\section{CONFLICT OF INTEREST}

The authors declare no conflict of interest.

\section{ACKNOWLEDGEMENTS}

This work was supported by EuroGentest, an EU-FP6 supported NoE, contract number 512148 (EuroGentest Unit 3: 'Clinical genetics, community genetics and public health', Workpackage 3.2).

1 Alter BP, Baerlocher GM, Savage SA et al: Very short telomere length by flow fluorescence in situ hybridization identifies patients with dyskeratosis congenita. Blood 2007; 110: 1439-1447.

2 Bessler M, Wilson BD, Mason PJ: Dyskeratosis congenita. FEBS Letts 2010; 584 3831-3838.

3 Calado RT, Young NS: Telomere Diseases. N Engl J Med 2009; 361: 2353-2365.

4 Dokal I: Dyskeratosis Congenita. Hematol Am Soc Hematol Edu Program 2011; 2011 : 480-486.

5 Du HY, Pumbo E, Ivanovich J et al: TERC and TERT gene mutations in patients with bone marrow failure and the significance of telomere length measurements. Blood 2009; 113: 309-316.

6 Fogarty PF, Yamaguchi H, Wiestner A et al: Late presentation of dyskeratosis congenita as apparently acquired aplstic anaemia due to mutations in telomerase RNA. Lancet 2003; 362: 1628-1630

7 Gramatges MM, Bertuch AA: Short telomeres: from dyskeratosis congenita to sporadic aplastic anemia and malignancy. Trans/ Res 2013; 162: 353-363.

8 Savage SA, Dokal I, Armanios $M$ et al: Dyskeratosis congenita: the first NIH clinical research workshop. Pediatr Blood Cancer 2009; 53 520-523.

9 Vulliamy TJ, Knight SW, Mason PJ, Dokal I. Very short telomeres in the peripheral blood of patients with X-linked and autosomal dyskeratosis congenita. Blood Cells $\mathrm{Mol}$ Dis 2001; 27: 3 53-77.

10 Vulliamy TJ, Marrone M, Knight S, Walne A, Mason PJ, Dokal I: Mutations in dyskeratosis congenita: their impact on telomere length and the diversity of clinical presentation. Blood 2006; 107: 2680-2685.

11 Walne AJ, Dokal I: Advances in the understanding of Dyskeratosis Congenita. Br J Haematol 2009; 145: 164-172.

Supplementary Information accompanies this paper on European Journal of Human Genetics website (http://www.nature.com/ejhg) 